Website: http://jsci.utq.edu.iq

Email: utjsci@utq.edu.iq زيادة التأثير السمي للمبيد البيرثرويدي سومسيدين Sumicidin باستخدام مستخلص بذور نبات

Tribolium ضد بالغات خنافس الطحين المتشابهة Pimpenella anisium الينسون . confusum

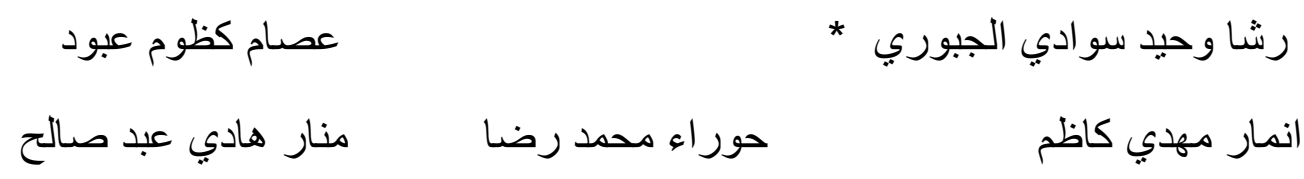

قسم علوم الحباة - كلية العلوم - جامعة بابل

الخلاصة

شملت الدراسة الحالية استخدام مستخلص بذور نبات الينسون لتتنيط المبيد البيرثريدي المصنع سومسيدين ضد خنفساء الطحين المنتابهة Tribolium confusum و ايجاد نسب القتل لتراكيز مختلفة من المبيد وحده ومزيج المبيد والمستخلص بنسب قتل مختلفة كذلك تم استخراج قيمة التزكيز نصف القاتل (Lc50 ) ونسب التنشيط لمعاملات المبيد

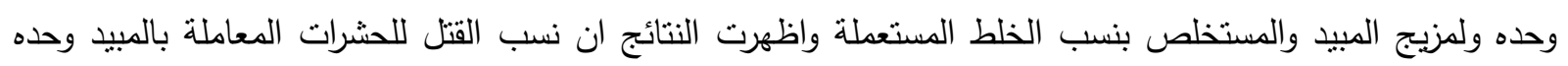
ازدادت بشكل ملحوظ عن نسب القتل للحشرات المعاملة بالمبيد وحده ازدادت بشكل ملحوظ عن نسب القبات القتل للحشرات

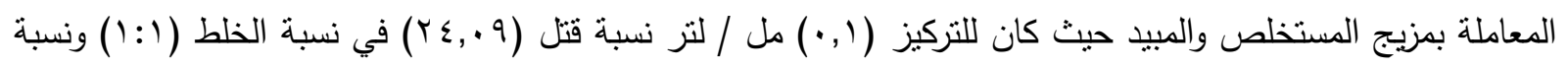

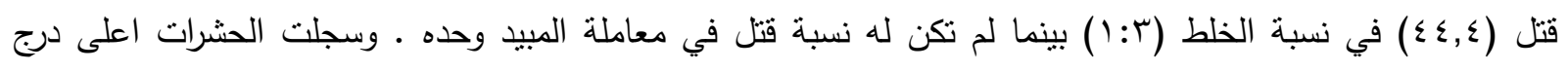

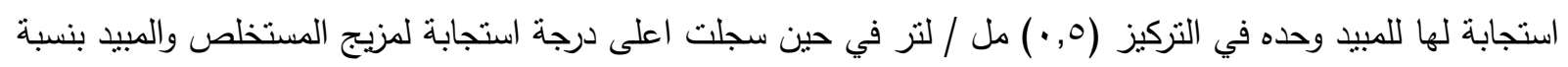

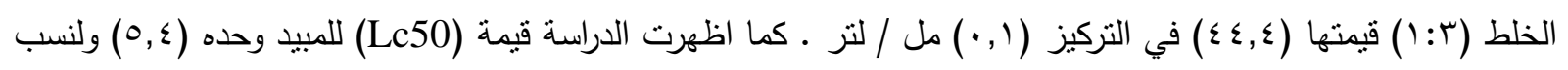

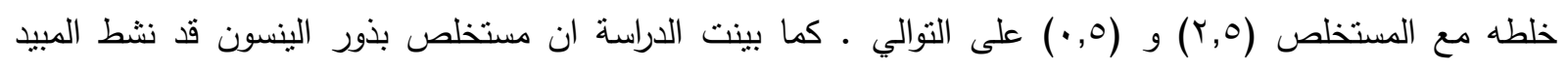

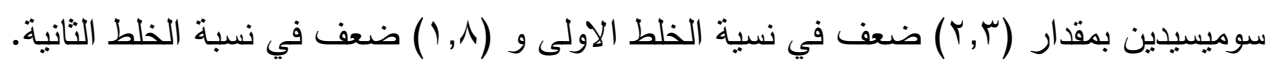

*Email : resha_mzj@yahoo.com 
كما ان نطور مقاومة سلالات مختلفة من افات المخازن

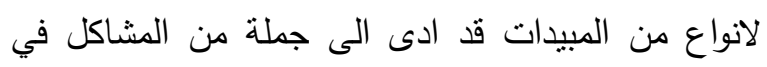
تخزين وتصنيع المواد الغذائية وخصوصا الحبوب ( Khalequzzaman and Khanom , 2006) هذا الصدد ذكر Collins (199.) ان مستويات المقاومة للمبيدات البيروثريدية سجلت اعلى مستويات لها لاى خنافس الطحين لذا فلابد من التفكير بطرق اخرى للسيطرة على حشرات المخازن ومنها خنافس الطحين فمن الممكن تتشيط المبيد البيروثرويدي بمنشطات غير سامة ومستساغة للانسان قليلة الكلفة المادية ومن صنع الطبيعة (Armitage , etal ., 1994) دمج المبيدات الكيميائية مع المستخلصات النباتية النباتية

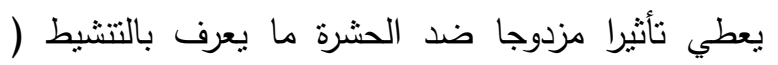
التآزر) Synergism بحيث يكون غير مؤذي للاغذية Khalequzzaman and Khanom , ) المخزونة هوني - $(2002$

درس الكثير من الباحثين تتشيط المبيدات باستخدام مواد

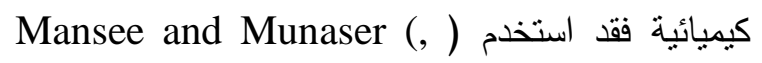
200) البابيرونيل بيدتوكسيد مع مبيدات الملاثيون والكلوربيريفوس - منيل ضد حشرة خنفساء الطحين الصدئية Tribolium castaneum كما تتاول باحثون تتشيط المبيدات البيرثرويدية بأستعمال

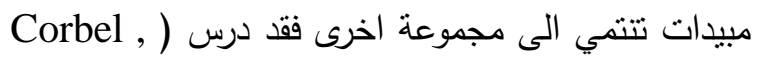
2003 ). etal النتشيط بين المبيد البيرثرويدي Permethrin الكارباميت(Carbamate) ضد يرقات بعوض الكيولكس وجرب Attia and Frecker (, 1984) دراسة التشيط باستخدام طيف الضوء Spectrum في سلالات خنفساء

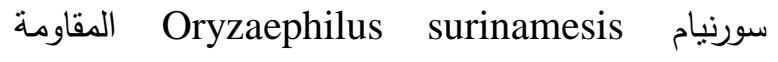
Organophosphorus (OP) لمبيدات من مجموعة ودرس(1987) . Bengston, etal تتشيط مبيدي ال

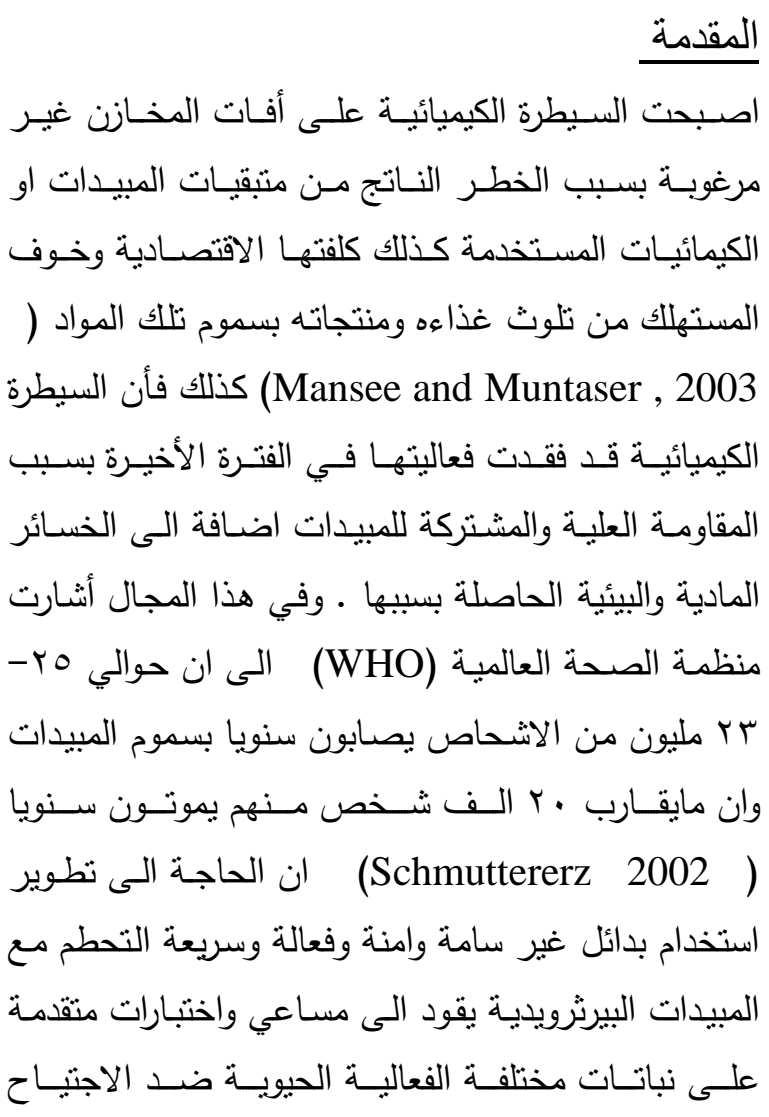
Okunlola et (الحاصل من افات المخازن invation 2008 , al وذللك لكونها مصـادر غنية بالمركبات الحيوية الفعالة مثل الفينولات والتربينات والقلويدات وغيرها (Ahn , etal ., 1998)

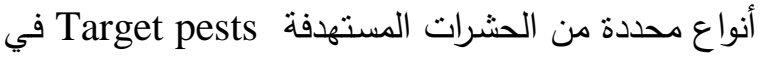
حين تكون مستسـاغة لـدى الإنسـان عند اختلاطهـا مـع غذاءه وسريعة التحطم لذلك فأنها تكون مناسبة الاستعمال الامر الذي يقود الى تطوير طرق جديدة وامنـة للسبطرة Eman , etal., 2009 ; ( ) على اصابات المخازن الغذائية Park , etal. , 2002 and Mansour, etal . , 2004) اضافة الى ان المستخلصات النباتية الحاوية على هذه المركبات يمكن ان تتحطم في البيئة بمعدل اسرع

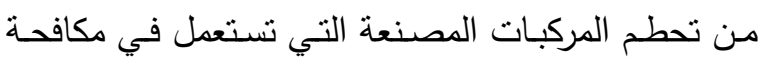
الافات (Moretti , etal ., 2002) ( 
(Less .) الطحين الحمراء الصدئية Tribolium castaneum وفي لاصي مazaq , etal ) مجال المنشطات الكيميائية استخدم 2006). المركب (PBO) لتشيط المبيد يد حشرة Fenpropathrin 2005 ) Abd el- Latif, etal . ودرس devastans ) تتثيط مستخلص بذور الكزبرة ببعض غازات الغلاف Micro - habitat Atmosphere gases الجوي ضد خنفساء الطحين وسوسة الرز خنفساء (MAS)

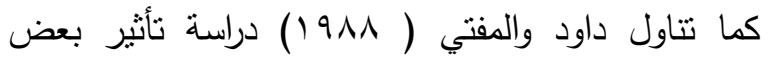
المنشطات على فتزة بقاء المبيدات المستخدمة ضد خنفساء سورينام Oryzaephilus surinamesis ، في حين

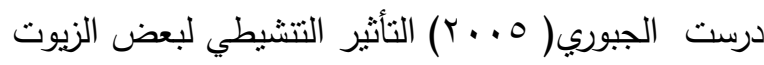
النباتية في المبيد كاراتي Karate ضد حشرة خنفساء الطحين المتثابهة Tribolium confusum واستعمل فئل مستخلص بذور الينسون اضافة الى مستخلص اوراق

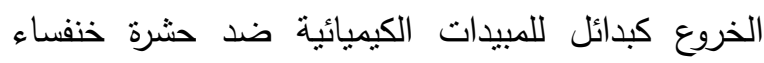
Log- الطحين المنتابهة حيث استخرجت خطوط سمية dose probit line

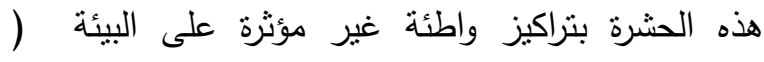

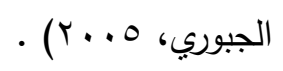

وفي مجال استعمال المستخلصات كمنشطات للمبيدات فقد درس Khalequzzaman and Islam (1992) التأثير التتشيطي لمستخلص أوراق وبذور نبات الداتورة في المبيد Methacrifos الدراسة الحالية الى استخدام المستخلص الكحولي لبذور نبات اليانسون Pimpenella anisium لزيادة الناثير السمي للمبيد الحشري Sumicidin عند استخدامة بتراكيز لئن منخفضة ومحاولة تقليل متنقيات هذا المبيد في البيئة واختزال تاثيرة على الاحياء الاخرى, يعد اليانسون من فلئ

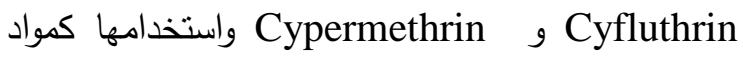
(1977) Desmarchelier , حافظة لحبوب القمح .ودرس تنشيط المبيدات البيرثرويدية ومبيدات الفسفور العضوية

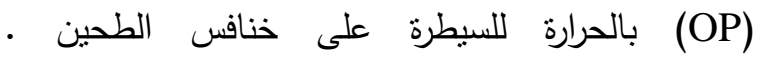
وحاول Koziol and Witkowski Permethrin \& التتشيط بالمزيج المزدوج لمبيدات Chorpyrifos \& وال Methyl parathirone ( على يرقات ثاقبة الذرة الاوربية ـ وبحث 1994 ). Merei , etal المشترك والتنشيط في بعض المبيدات البيرلاثرويدية ضد التدال

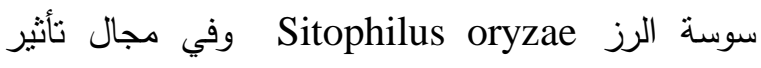
متبقيات المبيدات فقد جرب (Shawir , 1997 ) دراسة مجات

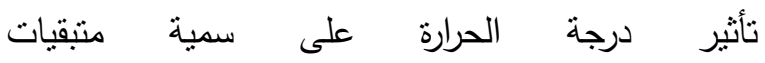
مبيدي Deltamethrin و اليرو دralomethrin في سوسة الرز وخنفساء الطحين وفي مجال كسر المقاومة لدى

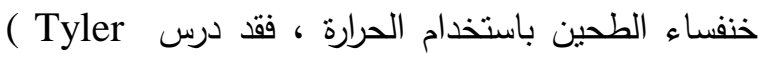
(and Binns ) نأثيرا لحرارة في ثمانية مييدات من مجموعة (OP) ضد سلالات مقاومة لحنفساء الطحين Tribolium castaneum وخنفساء سورينام - S . granaries, Oryzaephilus surinamesis وفي دراسة اخرى لاحظ . Watters , etal (1983)

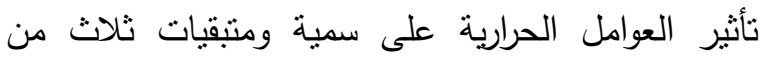
المبيدات البيرثريدية في خنفساء الطحين الصدئية

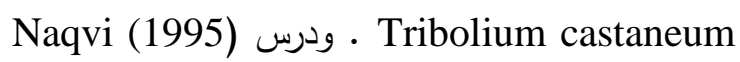

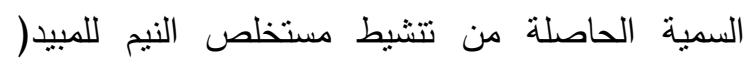
(Coopex 25 E . C المنزلية Meed and Filmer (وبين (C) domestica (1950) كيفية تتشيط مسحوق ال Ryania N- propyl والمركب Piperonyl cyclonene isomer Wedelia calendulacea التنثيطي لمستخلص نبات النير 
(r: (1) • استعملت ثلاث أطباق بتري بلاستيكي (1.5. 1.5 (8.5 × لكل تركيز وعملت فتحة في غطاء كل طبق

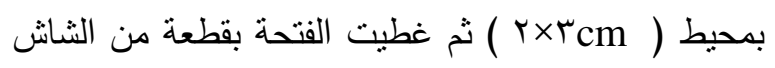

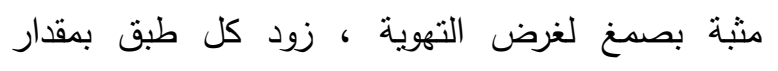

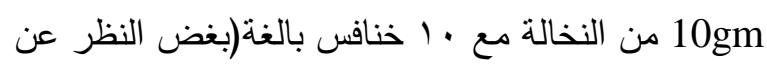

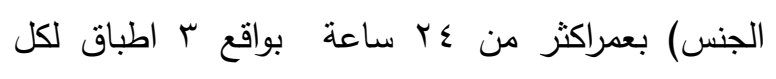
تركيز كذللك في أطباق السيطرة التي تحتوي على الني النخالة

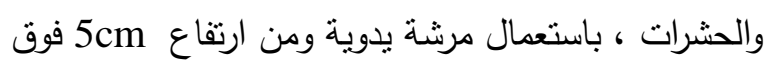

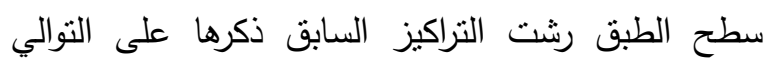

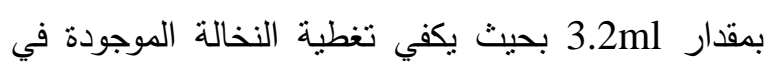

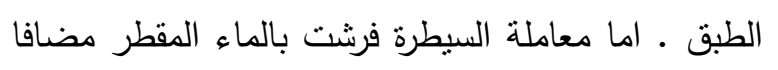

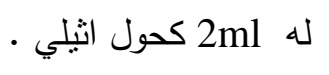

سجلت النتائج نسب هلالك البالغات بعد ع ك ساعة حضن لكل معاملة ثم عدلت النسب حسب معادلة ابوت ( Abott

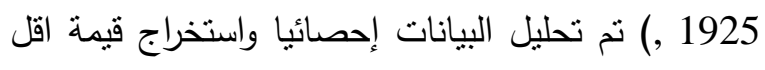
فرق معنوي LSD بالاستعانة بالنظام الإحصائي

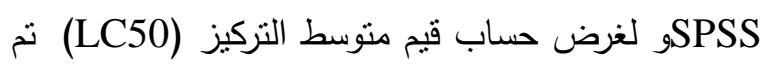
رسم خط السمية من خلال تحويل قيم التراكيز المستخدمة لتركية

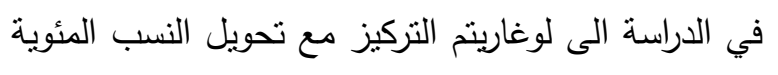

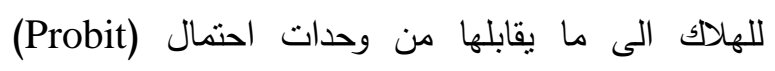

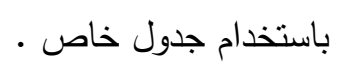

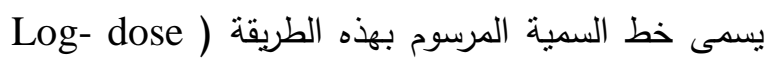
Log- باستخدام ورق خاص بسمى (probit line وللتأكد من دقة رسم خط السمية

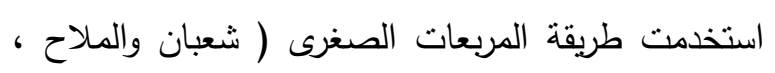

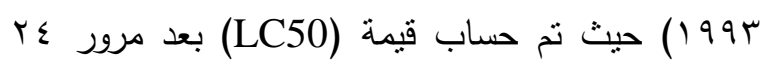

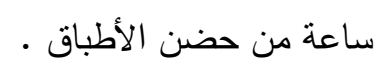
استخرجت نسبة التتشيط من خلال المعادلة الثالية : LC50

LC50 للمزيج (المبيد + المستخلص)
النبات الطبية الثائعة الاستعمال في محتلف المجالات

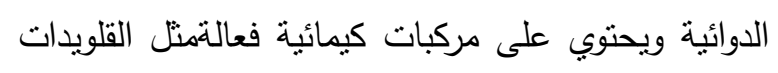

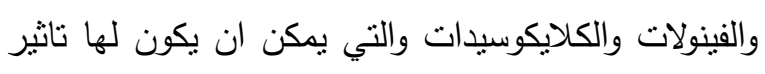

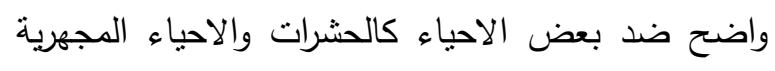
وبعض انواع القشريات(الراوي, ؟19 1). المو ادوطرق العمل

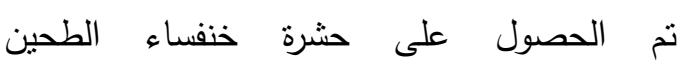
T.confusum من طحين مصاب اخذ من احد مخازن

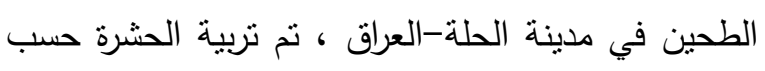

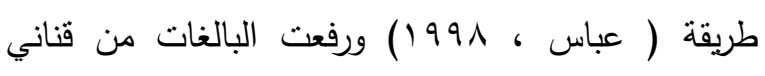

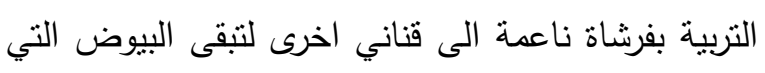

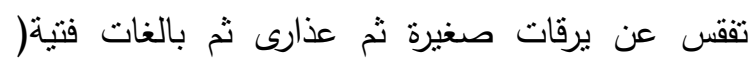

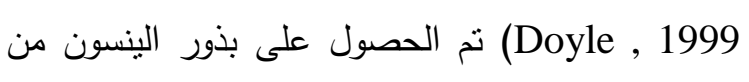

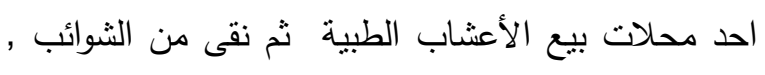

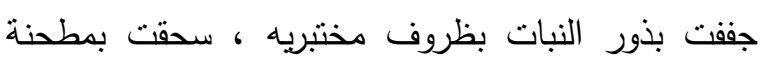
كهربائية حتى الحصول على مسحوق نباتي دقيق وخزن في الثلاجة لحين الاستعمال نم استخلاص المادة الفعالة

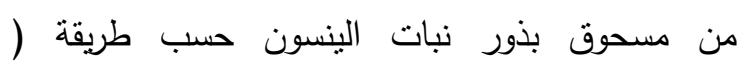

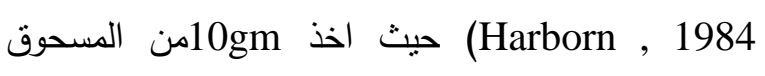

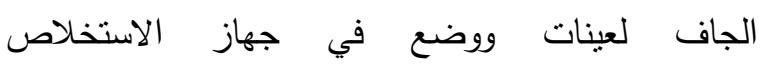
(السكسوليت) باستخدام المذيب (الكحول الاثيلي) بتركيز

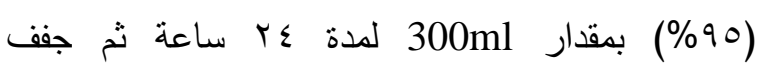

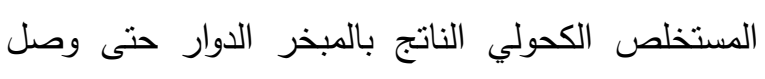

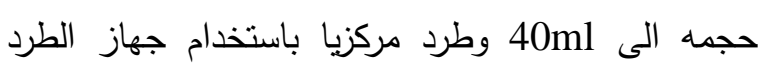

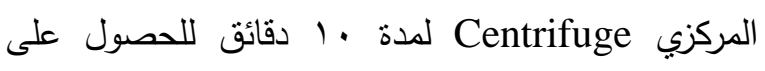
محلول أصلي Stock solution حضرث التراكيز ( ml/L سومسدين Sumicidin لوحدة ثم حضرت نفس التزاكيز

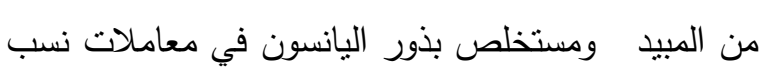
الخلط (1:1) وحضرت ثناث اضعاف التراكيز السابقة للمستخلص مع تثتيبت نفس تراكيز المبيد في نسبة الخلط 
المبيد وعند ذلك تكون الدراسة قد حققت الهدف في

استخدام تراكيز واطئة للمبيد بتتشيطه بمواد غير سامة وغير مكلفة اقتصاديا بالإضافة إلى كسر مقاومة الحشرة للمبيد باستخدام المستخلص • يتضح من الثكل رقم (1)

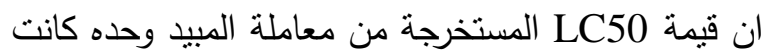
( ) مزيج المستخلص والمبيد (r,r) و (, (•) لنسب الخلط على التوالي كما في الاثكال (r) و (r) ومن خلآ هذه الاشكال استخرجت نسبة النتشيط حيث بلغت قيمتها في نسبة الخلط (r:1) كانت (^,^•(1) وهذا يعني ان مستخلص بذور الينسون قد تتشيط عمل المبيد سومسيدين وخصوصا في التراكيز الواطئة في نسبة الخلط الاولى بلى بمقدار (Y,r) ضعف سمية المبيد وحده وفي نسبة الخلط الثانية بمقدار (,^) ضعف .تعتقد الدراسة ان قابلية المنشط المستخدم (مستخلص بذور الينسون) على تتشيط المبيد سومسيدين قد اعتمدت واحدة او اكثر من ميكانيكيات التشنيط المعروفة ، فقد يكون المنشط قد قلل من ايض المبيد داخل جسم الحشرة وتفككه او تحلله المائي الى مركبات غير سامة كما هو الطبيعي في فسلجة جسم الحشرة الداخلية وبذللك بقي محافظا على قدرته السمية وزيادة السمية (Baki etal ., 2005 ) بينما بذكر Otaki and Williums ( 1970 ) يحتوي على انزيمات قادرة على تحطيم الهرمونات مثل هرمون الانسلاخ (MH) الذي يمثل احد ميكانيكيات عمل Hewlett (1960) المستخلص النباتي • في حين يذكر ان المستخلص كمنشط يعمل على تنثيط الانزيمات المزيلة لسمية المبيد . ومن الدراسة الحالية يمكن الاستتناج بأنه يمكن زيادة فعالية المبيد الحشري Sumicidin بتراكيز

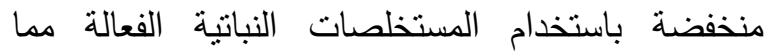
يضمن عدم وجود منتقيات سامة قد تؤثز على الأحياء الأخرى وتنبب تلوث البيئة.
اظهرت نتائج التجربة(جدول () ان التراكيز الواطئة من المبيد سومسدين ا،، و 0Y، ، سمالتز لم تسبب قتل لبالغات الحشرة تحت الدراسة الى ان التراكيز العاليةه، .

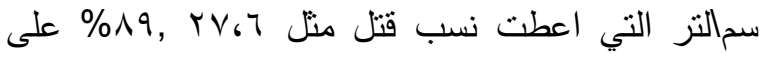
التوالي. كما يلاحظ من النتائج ان اضافة مستخلص بذور اليانسون سبب زيادة في نسب القتل حتى في التراكيز الواطئة. تحتمل الدارسة ان يكون سبب سمية المستخلص المعامل به هو وجود مركبات مختلفة تؤثر على الفعاليات الحيوية للحشرات المختبره وهذا يتفق مع بـ ماذكره (Jbilon etal ., 2006 ) بأن المركبات الكيميائية النباتية من مجموعة Ajuga species المسؤولة عن سمية هذه النباتات وبالتالي تاثيرها على الفعاليات الحيوية للحشرة. او قد يكون المبيد قد تعرض الى عملية ايض تنشيطي في داخل جسم الحشرة بنفاذه من خلال الكايتين المغلف لجدار الجسم بفعل احد ميكانيكيات التشتيط مما جعل سميته تزداد (Fahmy , 2008) او قد تكون كمية المبيد التي وصلت الى الموقع الحساس في الحشرة بفعل التأثير التشيطي للمستخلص كانت قد وجدت درجة تقبل عالية لها في الموقع الحساس في جسم الحشرة حسبما ذكره( شعبان و الملاح ، ب99 1 ) .يتضح من الجدول رقم (Y) ان درجة استجابة الحشرة لمزيج مستخلص بذور الينسون والمبيد سومسيدين والتي هي حاصل الفرق في نسبة القتل بين تركيزين متتابعين (عبد الحميد و عبد المجيد ، 911 (91) بنسب الخلط المذكورة أخذت بالتزايد بزيادة نسبة الخلط وبالتراكيز الواطئة وهذا يعني ان المبيد المستعمل قد تتشط عمله بالمستخلص واصبح اكثر سمية ضمن تراكيز كانت غير سامة للحشرة قبل تتشيطه وهذه النتيجة كانت منوافقة مع ماتوصلت اليه (الجبوري

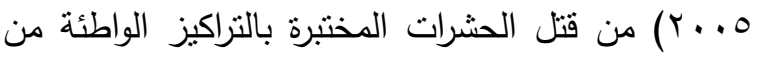

جدول( () : العلاقة بين تراكيز مختلفة للمبيد سومسدين Sumicidin بمفرده ومزيج $\checkmark \cdot$ المبيد ومستخلص بذور الينسون بنسب الخلط (1:1) و (r:1) ومعدل نسبة القتل 
جدول(ץ): درجة استجابة خنافس الطحين المتشابهة T.confujum لتراكيز مختلفة

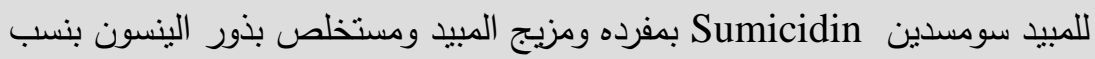

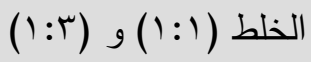

\begin{tabular}{|c|c|c|c|}
\hline لثرجةُ الاسنَجابةُ & للمزيجة الأسئجابةً & 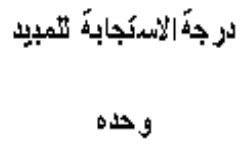 & |تؤر كيز (من) /لائر) \\
\hline 44.4 & 24.09 & 0 & 0.1 \\
\hline 0 & 10.31 & 0 & 0.25 \\
\hline 37 & 17.3 & 27.6 & 0.5 \\
\hline 14.8 & 41.37 & 62 & 1 \\
\hline
\end{tabular}




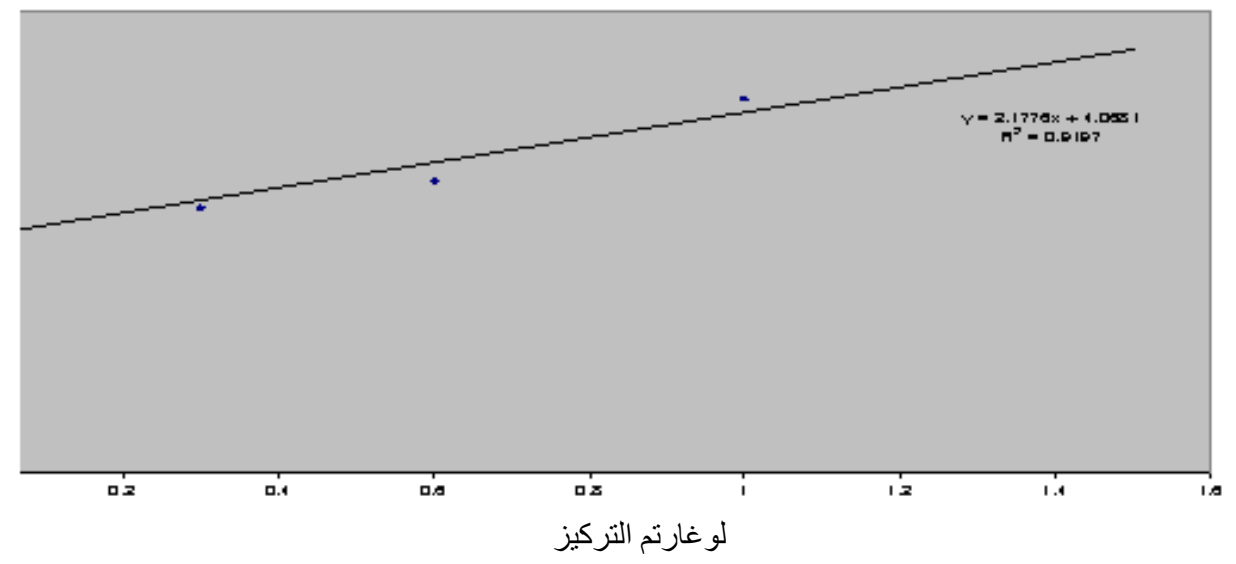

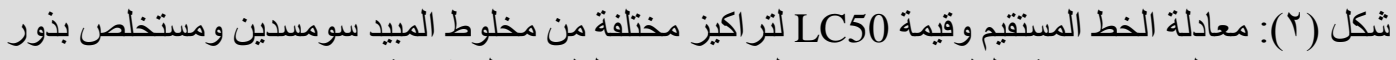

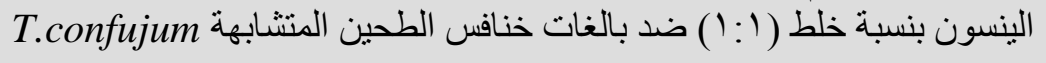

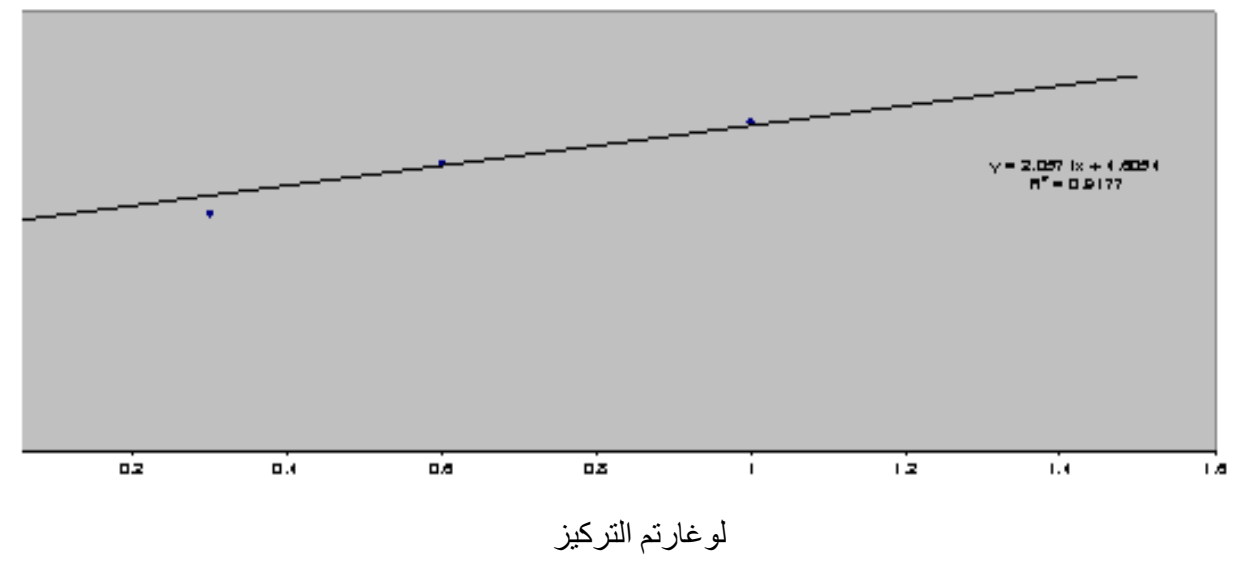

شكل (r): معادلة الخط المستقيم وقيمة LC50 لتر اكيز مختلفة من مخلوط المبيد سومسدين ومستخلص بذور

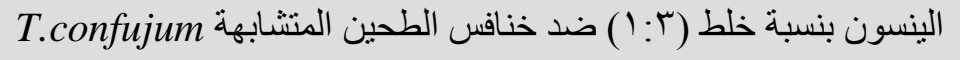

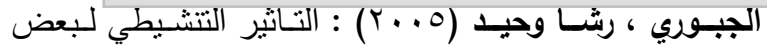

الزيـوت النباتيـة في المبيـد البيرثرويـدي Karate ضــ 
pests . Egypt . J Agric . Res ., 83 (3) , 2005

Ahn Y. J . , Lee H . S. and Kim G . H . , (1998)

: Insecticidal and A caricidal Activity of Caracole and B-Thujaplicine derived from Thujopsis dolabrata var. Honda Sawdust .J . chem. . Ecol.,24 (1): 81-90 .

Armitage , D . M . , P. M . Cogan and D .R . Wilkin,(1994): Integrated pest management in stored grain : Combining suface insecticide treatment with aeration . Journal of stored products Research , 30 (4) : 303-319 .

Attia , F .I . and T . Frecker (1984) : Crossresistance spectrum and synergism studies in organophosphorus resistant strain of Oryzaephilus surinamesis L . in Australia . Journal of Entomology , 77 (3): 13671370 .

Baki , M . A . ; AKhtar , N . ; Rahman , M . M . ; Islam , M . N . ; Hossain , M .; Islam , N. ; Alam , M . K . ; Islam , R . ; Khatun, N . A . and Mondal , K . (2005) .Synergistic action of Wedelia calendulacea (Less .) plant extracts with Lambda Cyhalothrin on adult red flour beetle Tribolium castaneum (Herbst) . J ournal of Agronomy, 4(1) : 18-22 (2005) .

Bengston , M . , J . M . Desmarchelier , B . Hayward , R . Henning , J . H . Moulden, R . M . Noble, G . Smith , J . T . Snelson, R . Sticka, S . Thomas, B . E . Wallbank , and D . J . Webley . 1987 . Synergized Cyfluthrin and Cypermethrin as grain protectants on bulk wheat, pesticides , 21(1): 23-37.

Bengston , M .; R . A . H . Davies ; J . M . Desmarchelier ; R . Henning ; W . Murrey ; B . W . , Simpson ; J . T . , Snelsson ; R . Sticka and B . E . Wallbank.(1983):

Organophosphorothioates and synergized synthetic pyrethroids as grain prpotectants on bulk wheat . Pesticides Science , 14 (2): 373-384 .

Coollins , P .J . (1990) : A new resistance to py rethoids in Tribolium castaneum Pesticides Science, 28(1) : 101-115.

Corbel , V . ; chandre , F . ; Darriet , F . ; Lardeux , F. and Hougard , J-M .,

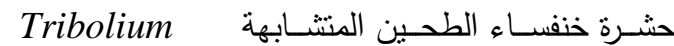

confusum • رسالة ماجستير - كلية العلوم - جامعة الطن

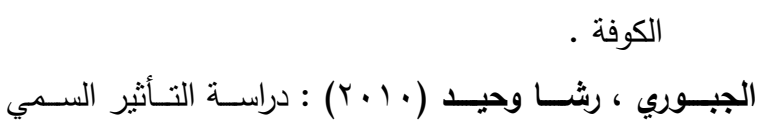

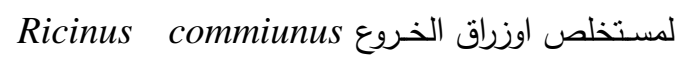

وبذور الينسون Pimpenella anisium في بالغات

خنافس الطحين المتشابهة Tribolium confusum

. (Coleoptera : Tenebrionidae) (duVal .

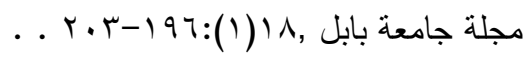

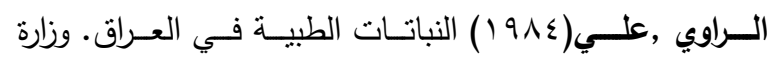

$$
\begin{aligned}
& \text { الزراعة-بغداد ,العراق. }
\end{aligned}
$$

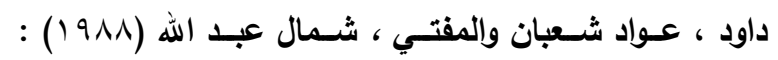

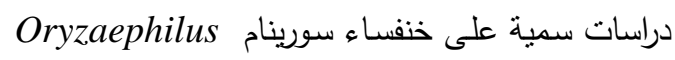

$$
\begin{aligned}
& \text { surinamesis }
\end{aligned}
$$

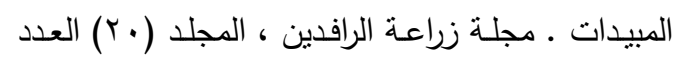

$$
\begin{aligned}
& \text {. 1911 ، (1) } \\
& \text { شعبان ، عواد والملاح ، نزار مصطفى (ب991 (1) : المبيدات . } \\
& \text { وزارة التعليم العالي والبحث العلمي، مؤسسة دار الكتب لمعي } \\
& \text { للطباعة والنشر / جامعة الموصل. } \\
& \text { عباس ، سـهلة حورشيد (991) ) : دراسـة تأثثر اربع نباتات } \\
& \text { عشبية على حشرة خنفسـاء الطحين الحمراء الصدئية } \\
& \text { Coleoptra) Tribolium castaneum (Herbst) } \\
& \text { لات Tenebrionidae } \\
& \text { للبنات -جامعة نكريت. } \\
& \text { عبد الحميـد ، زيـدان هنـدي وعبد المجيـد ، محمـد ابـراهيم }
\end{aligned}
$$

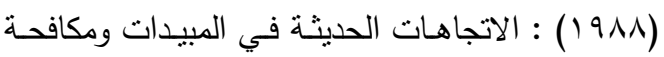

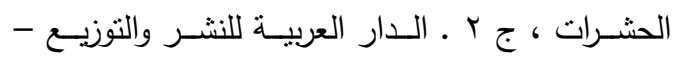

$$
\begin{aligned}
& \text { القاهرة . }
\end{aligned}
$$

Abbott, W .S . (1925) . A method of computing effectiveness of an insecticide . J. Econ . Entomol . 18 : 265-267.

Abo El- Latif , A . M , A . E . Abo El-Aziz and M. S.Gharib .( 2004 ) Toxicity and joint action of coriander seed extract and modified micro - habitat atmosphere gases (MAS ) against some stored grain insect 
Tribolium castaneum Tribolium in for. Bull ., 32: 27-78

Koziol , F . S . and J . F . WitKowski . 1982 . Synergism studies with binary mixtures of permethrin plus methyl para thion, chlorpyrifos, and malathion on European corn borer Larvae . Journal of Economic Entomdogy . 75 (1) : 23-30

Mansee , P . H and Montasser, M. R . (2003) : Maximizing toxicity of certain insecticides against Tribolium castaneum. Agriculture and Marine Sciences , 8 (1): 27- 34 (2003)

Marei , A . M . Khattab, and M . R . Montasser . 1994 Toxicity, joint toxic effect and synergism of certain pyrethroids against the rice weevil, Sitophilus oryzae . Alexandria journal of agricultural Research , 39 (3) : 347-362

Mondal , K . A . M . S . H . 1990 . Combined action of methyl quinine, aggregation pheromone and pirimiphosmethyl on Tribolium castaneum larval mortality . Pakistan J. Zool . , 22(3) : 249-255

Moretti , Mario D. L .; Sanna - Passino , Giovanni ; Demontis , Stefania and Bazzoni , Emanuela (2002) : Essential oil for mutations useful as a new tool for in sect pest control . AAPS pharm sci tech 3 (2) article $13: 1-6$ (2002)

Nagvi , S . N . H . , Jahan , M ., Tabassum , R . , Qamar , S . J . \& Ahmed , I . (1995) . Toxicity and teratogeny caused by coopex $25 \mathrm{E}$. C . and aneem extract (N-7) against $3^{\text {rd }}$ in star larvae of Musca domestica .Pakistan J . Zool ., 27 (1) : 27-31

Okunlola A . I . ; Ofuya T . I. and Aladesanwa R . D . (2008) : Efficacy of plant extracts on major insect pests of selected leaf vegetables in south western Nigeria . Agricultural journal , 3(3) : 181184,2008

Otaki , T . and C . M . Williums , (1970) . Interaction of $\beta$ - ecdysdon and cyasterone by larvae of the Fresh fly, sarcophagi peregrine and pupa of the silk worm, Samia Cynthia . Biol. Bull . Marine Biol . lab . woods Hole, 138: 326-333

Park , B. S. ; Lee, S . E . ; Choi , W . S . : J eong, C. Y . ; Song, C . and Cho, K . Y
(2003) : Synergism between permethrin and propoxure against Culex quinquefasciatus mosquito larvae. Medical and Veterinary Entom ., 17(2) : 158.2003

Desmarchelier , J . M .( 1977) . Selective treatments, including combinations of pyrethroid and organophosphorus insecticides for control of stored product Coleoprera at two temperatures . Journal of stored products Research , 13 (2) : 129-137.

Doyle , D . , (1999) . Culturing confused flour beetles (Tribolium castaneum ). Arizone Dendrobate Ranch .

Emam , Ahmed M .; Swelam , Emam S . and Megally , Nadia Y . , (2009) : Furocou marin and Quin olone Alkaloid with . Larvicidial and Antifeedant activities Isolated from Ruta chalepensis leaves . $J$. Natural products . , 2: 10-22 (2009).

Fahmy , A . R . (2008) . Role of carboxyamidase in the in vivo metabolism of chlorfluazuron in the Black Cutworm Agrotis ipsilon (Hfn) ( Lepidoptera : Nuctidae ). Egypt Acad. J . Biology . Sci . , 1 (2): 65-70 (2008)

Harborn J - B . (1993) : Introduction to Ecological Biochemistry . $4^{\text {th }}$ edition Academic press . New York

Hewlett , P . S . , 1960 . Joint action in Pesticides in Analyses of pest control Research ( Metcalf, R . L . Ed .) J. Wiley and Sons . Inc . Ny . , PP : 27-74

Jbilou,R. ;Ennabili , A. and Sayah , F. (2006).:Insecticidal activity of four medicinal plant extracts against Tribolium castaneam (Coleoptera:Tenebrionidae).African Journal of Biotechnology Vol.5(10),PP.936-940,16 May 2006.

Khalequzzaman , M . and Sultana , S . (2006) Insecticidal activity of Annona squamosa L. Seed extracts against the red flour beetle, Tribolium castaneum . J . bio - sci , 14 : 107-112, 2006

Khalequzzaman , N . and Islam , M . N (1992) Synergism of Datura metel (L .) leaf and seed extract with Methacrifos on 
Tribolium castaneum . Alexandria Journal of Agricultural Research , 42(3) : 207-215

Tylar , P . S . and T . J . Binns .( 1982 ). The influence of Temperature on the susceptibility to eight organophosphorus insecticides of susceptible and resistance strain of Tribolium castaneum, Oryzaephilus surinamesis, Sitophilus granaries . Journal of stored products Research, 18 (1) : 13-19

Watters , F . L . , N . D . G . White, and D . Cote .( 1983 ) Effect of temperature on to Toxicity and resistance of 3 Pyrethroid insecticides applied to fir plywood for the control of the red Tribolium castaneum ( Coleoptera :Tenebrionidae) . Journal of Economic Entomology, 76 (1) : 11-18

Willkison, C. F. (1971) Insecticides synergists and their mode of action. Proceedings of the $2^{\text {nd }}$ International IUPAC congeries of pesticides chemistry $2: 117-159$
. , (2002) :Insecticidaland Acaricidal activity of pipernonaline and piperoctadecalidine derived from dried fruits of Piper longum L . Crotection, 21: 249-251

Razaq , M . ; Aslam , M . and Suhail , A.( 2006 ). Synergism of pyrethroids with piperonyl butoxide (PBO) in assid, Amrasca Devastans (Dist) ( Homoptera : Cicadellidae ) from Pakistan . Pak. Entomol ., 28 (2), 2006

Reed , R . P . \& Filmer, R . S . (1950). Activation of ryania duct by piperonyl cyclonene and N-propyl isomer . J . Econ . Entomol , $43: 161-164$

Schmutterer, H . ( 2002) The Neem Tree, Azadirachta indica A .Juss and Othermeliaceous plants Sources of Unique Natural products for Integrated pest management medicine and other purposes . Mumbia , India

Shawir , M. S. and A. H. Mansee (. 1997) . The influence of temperature on the residual toxicity of deltametherin and tralomethrin to sitophilus oryzae and

\title{
Maximizing toxicity of pyrethroid insecticides Sumicidin by using anis seed extracts Pimpenella anisium as a synergist against adult of confused flour beetles Tribolium confusum.
}

\begin{abstract}
This study deals with using of anis seeds extracts to synergy the synthetic pyrethroid insecticide Sumicidin against confused flour beetles Tribolium confusum. Also found percents of mortality to different concentrations of insecticides alone and mixture with extract by different mixture ratios. Lethal concentration of 50(Lc50) value was concluded then synergistic ratio for sumicidin alone tests and the using ratio tests mixture too. Results show that mortality ratio of insects exposed to sumicidin alone were increased more than to mixture ,so the mortality ratio of the concentration $(0.1) \mathrm{ml} / 1$ as $(24.09)$ in the mixture ratio (1:1) and (44.4)in (1:3)for the same concentration while there was no mortality ratio in sumicidin alone test .Experimented insects were recorded the highest responsible degree to sumicidin alone in $(0.5) \mathrm{ml} / \mathrm{l}$ concentration while ahigh degree for the mixture of sumicidin and extracte was in $(1: 3)$ as $(44.4)$ in $(0.1) \mathrm{ml} / \mathrm{l}$ concentration .Also study was showed the Lc50value for sumicidin alone (5.4)and for mixtures ratios (2.5) (0.5) respectively .Study showed that the anis extract was synergetic sumicidin as(2.3)fold in the first mixture ratio and (1.8)fold in the second mixture ratio.
\end{abstract}

